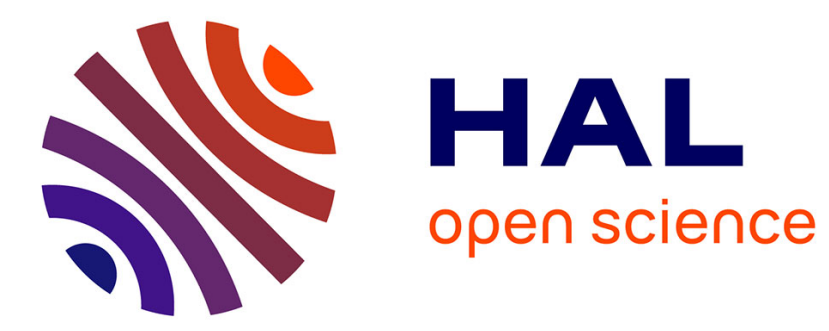

\title{
Morphological characterization of a polymeric microfiltration membrane by synchrotron radiation computed microtomography
}

Jean-Christophe Remigy, Martine Meireles, Xavier Thibault

\section{- To cite this version:}

Jean-Christophe Remigy, Martine Meireles, Xavier Thibault. Morphological characterization of a polymeric microfiltration membrane by synchrotron radiation computed microtomography. Journal of Membrane Science, 2007, 305 (1-2), pp.27-35. 10.1016/j.memsci.2007.06.059 . hal-00323055

\section{HAL Id: hal-00323055 \\ https://hal.science/hal-00323055}

Submitted on 19 Sep 2008

HAL is a multi-disciplinary open access archive for the deposit and dissemination of scientific research documents, whether they are published or not. The documents may come from teaching and research institutions in France or abroad, or from public or private research centers.
L'archive ouverte pluridisciplinaire HAL, est destinée au dépôt et à la diffusion de documents scientifiques de niveau recherche, publiés ou non, émanant des établissements d'enseignement et de recherche français ou étrangers, des laboratoires publics ou privés. 


\title{
Morphological characterization of a polymeric microfiltration membrane by synchrotron radiation computed microtomography
}

\author{
J.C. Remigy $^{(1)}$, M. Meireles ${ }^{(1)}$, X. Thibault ${ }^{(2)}$ \\ (1) Laboratoire de Génie Chimique, Université Paul Sabatier, CNRS UMR 5503 \\ 118 Route de Narbonne, 31062 Toulouse cedex 4, France. \\ (2) ESRF, 6 rue Jules Horowitz, BP220, 38043 Grenoble cedex, France.
}

\begin{abstract}
Most commercial polymeric membranes are prepared by phase inversion. The performance of the membranes depends greatly on the morphology of the porous structure formed during the different steps of this process. Researchers in this field have found it extremely difficult to foresee how a change in the composition of the polymer solution will affect pore formation without a set of methods designed to yield detailed knowledge of the morphological structure. This paper reports the new potential associated with X-Ray synchrotron microtomography to characterize the 3D structure of a PvDF hollow fibre microfiltration membrane prepared by phase inversion. 3D morphological data obtained from the ID19 line at the ESRF are presented. The membrane actually appears as a complex three-dimensional bi-continuum of interconnected pores. Within the hollow fibre structure, different regions with various thicknesses and pore size distributions have been identified and well characterized. Transversal views show the anisotropic finger-like structure of pores, while longitudinal sections reveal a honeycomb structure which resembles the structure of highly concentrated water in oil emulsion or dispersion. This typical structure might be obtained during the phase inversion process. How the phase inversion process may result in these morphologies is finally discussed.
\end{abstract}


Keywords: phase inversion - hollow fibre microfiltration membrane - pore-formation - X ray synchrotron microtomography

\section{Introduction}

The development of membrane technology in separation processes has been continuously expanding in the recent decades. A large number of membranes have been developed and used in different applications. Polymeric membranes have been developed for a variety of industrial applications including microfiltration, ultrafiltration and reverse osmosis. Each application imposes specific requirements on the membrane material and pore structure. The final morphologies of polymeric membranes vary greatly depending on the processing conditions used for manufacturing. Most commercial polymeric membranes are prepared by a phase inversion process. In this process, a concentrated homogeneous polymer solution is coagulated by a change in composition. The change can be achieved either by introducing new species or selectively removing others. This is generally accomplished by immersion of the polymer in a bath that is solvent miscible but non-solvent for the polymer. The nonsolvent begins to diffuse into the polymer solution and, concurrently, the solvent begins to diffuse into the coagulation bath, while the polymer diffuses very little because of its low mobility. These simultaneous phenomena cause the polymer solution to decompose into two phases (i.e. unstable thermodynamic state): a polymer-rich phase and a polymer-poor phase. At a certain stage, the polymer-rich phase becomes a gel and / or a solid while the polymerpoor phase develops into pores. The performance of the membrane depends largely on the morphology produced during this phase separation.

From a theoretical point of view, the thermodynamic basis of phase transition is well known and some mass transfer models in $1 \mathrm{D}, 2 \mathrm{D}$ and 3D have been made to understand the kinetics of the process before phase separation occurs [1]. Such approaches are based on the free 
energy function and phase diagram for the non-solvent /solvent/ polymer ternary system. Still, the exact route of the development of pores during phase separation and solidification has not been fully described.

From a practical point of view, there are some concerns about the preparation of the concentrated polymer solution, which has also been overlooked as a parameter capable of affecting membrane morphology by the presence of sizable inclusions or defective structures [2]. A significant number of experimental research works have therefore focused on the preparation of defect-free asymmetric polymeric membranes. Researchers in this field have found it extremely difficult to detect and interpret such defects in the final material in terms of physicochemical processes having occurred during the processing of the polymer solution, just as it is also extremely difficult to foresee how a change in the composition of the polymer solution will affect the pore morphology.

Although it can be argued that achieving better control of the phase inversion process is a long way off, one has also to consider that this is only achievable through a set of methods designed to yield detailed knowledge of the morphological structure of a membrane.

Traditionally, indirect, invasive methods such as scanning electron microscopy (SEM or FESEM), have been used to characterize membrane morphology [3, 4]. However, Scanning Electronic Microscopy techniques, although capable of estimating some features at high resolution, suffer from the drawbacks the structure potentially being damaged during the drying involved in sample preparation and hence do not provide accurate estimates of the structure. SEM techniques are also limited to flat surfaces, a distinct disadvantage for the complete observation of a polymeric hollow fibre in its original shape. Microscopic techniques also require a very large number of observations to give good statistics.

Yeo [4] used XMI to observe the fouling phenomena involved during the filtration of hydroxide iron particles. Projection images of the internal structure of the membrane were 
obtained only when the membrane was dry due to the relatively low used X ray energy (10 $\mathrm{keV})$.

In this context, the use of modern synchrotron X-ray radiation sources provides several new possibilities. Since their discovery, X-rays have been used to image the bulk of materials that are not transparent for visible light by taking advantage of their inhomogeneous absorption.

Tremendous progress was made when 3D display became available with computer assisted tomography. From the many two-dimensional (2D) images (radiographs) recorded at various angular positions of the objects and via appropriate algorithms (described for instance in reference [5]) and the derived software, 3D data volume, projections or perspective renditions of the objects can be obtained at will. Microtomography with spatial resolution better than $20 \mu \mathrm{m}$ has recently emerged with bench scale X-ray tomographic instruments. However, the best quality images in terms of signal-to-noise ratio and spatial resolution are still obtained on instruments located in synchrotron radiation facilities which could use a parallel monochromatic X-ray beam.

This paper reports the new possibilities associated with the availability of third generation sources of SR, such as ESRF, to characterize the 3D morphology of a PVDF hollow fibre microfiltration membrane prepared by phase inversion

\section{Materials and methods}

\subsection{Membranes}

The polymer solution was prepared by mixing polyvinyldifluoride co hexafluoropropylene (PvDF co HFP HF) (Solef 21216/1001, Solvay Bruxelles) with lithium chloride (Aldrich), dimethylacetamide (Aldrich) and water. The composition of the collodion is given in Table 1 . Water was used as a non-solvent of the polymer. 
Hollow fibre microfiltration membranes were spun at room temperature using the dry/wet phase inversion method which has been described elsewhere [6]. Table 1 gives the spinning conditions of the hollow fibre used in this paper. The nascent hollow fibre emerged from the tip of the spinneret and passed through an air gap $(500 \mathrm{~mm})$ before entering the coagulation bath. The hollow fibres extruded from the spinneret at a speed of $3.4 \mathrm{~m} \cdot \mathrm{min}^{-1}$ fell freely into the coagulation bath. The prepared hollow fibre membranes were stored in the water bath for $72 \mathrm{~h}$ at room temperature to remove the additive and then kept in a sodium bisulphite solution at $7^{\circ} \mathrm{C}$ to prevent collapse of the structure and microbial growth.

Membrane modules were prepared to test membrane performance in terms of pure water permeation flux. The measured pure water permeation flux was $3.8 .10^{-8} \mathrm{~m}^{3} \mathrm{~m}^{-2} \mathrm{~Pa}^{-1} \mathrm{~s}^{-1}$ at $25^{\circ} \mathrm{C}$ or $230 \mathrm{~L} \mathrm{~h}^{-1} \mathrm{~m}^{-2} \mathrm{bar}^{-1}$ at $25^{\circ} \mathrm{C}$. From SEM images of the external surface, the measured external pore size range was between 0.07 and $0.37 \mu \mathrm{m}$ with the mean pore size of about $0.17 \mu \mathrm{m}$. The internal pore size was estimated to be below $50 \mathrm{~nm}$ from FESEM images.

\subsection{Structure and morphology of membrane}

\subsubsection{SEM technique}

The morphologies of the cross-section and the external surface of the hollow fibre membrane were observed with a scanning electron microscope (SEM, CM_INSTRUMENT JSM 6700F,

JEOL, Japan). After the solvent exchange step, the hollow fibre membrane samples were cryogenically fractured in liquid nitrogen and then coated with gold to obtain an adequate contrast.

\subsubsection{Synchrotron radiation computed microtomography (SR $\mu C T$ )}

The morphologies of the hollow-fibre membrane were characterized through synchrotron radiation computed microtomography at the European Synchrotron Radiation Facility, Grenoble (France) on the ID 19 beamline [7]. Computed microtomography (SR $\mu C T$ ) consists in recording a number of projections (radiographs) of the object at different angles (from 0 to 
$180^{\circ}$ ) and reconstructing a 3D image from these projections with the help of a suitable algorithm.

The European Synchrotron Radiation Facility offers access to very high intensity of the X-ray beam, For ID19, the tremendous characteristics of the electron beam lead to a source size of 30 microns vertically and 120 microns horizontally with a high brilliance and a high lateral coherence). The availability of a suitable detector simultaneously providing a large dynamic range and low noise with a transfer time lower than the exposure time (Fast Readout Low Noise FRELON Camera developed at ESRF - 2048 x 2048 pixel and 14 bits [5, 8]) is also a marked advantage of this facility.

Compared to a standard X-ray tube, the SR source and used technology allows reducing the exposure times and improving the signal-to-noise ratio. Therefore the quality of the images acquired using the ID 19 SR microtomography set-up at ESRF is better than standard bench $\mathrm{X}$-ray tomograph. Details can be found in references $[5,7]$.

The monochromatic parallel beam also available at ESRF on beamline ID19 has many advantages. Among them, reconstruction algorithms that are exact - that is to say free from approximations or reconstruction artefact thus quantitative measurements are possible. One drawback of this is that no magnification can be obtained from the detector, i.e. spatial resolution mainly results from the effective pixel size of the detector. The increase of the spatial resolution while keeping the same signal-to-noise ratio requires a dramatic increase in the number of photons or exposure time. As already pointed out, the availability of intense parallel, monochromatic beams makes this possible with a lower limit for the resolution of about $0.28 \mu \mathrm{m}$ (mostly determined by CCD camera with suitable optics). The monochromaticity of the beam is the key point also since it is the basic condition which enables quantitative measurement of the absorption coefficient $\mathrm{E}(\mathrm{x}, \mathrm{y}, \mathrm{z})$ and opens up the 
feasibility of mapping density using suitable calibration. Thus the interest shifts from the architecture of the sample to the densitometry of the solid parts.

Hence, a clear advantage of microtomography using synchrotron radiation is the possibility of a quantitative evaluation [5].

The aim of a tomographic investigation is to recover the 3D information. Radiographic projections of the samples were measured at different angles and stored digitally. The image visualisation of the $3 \mathrm{D}$ reconstructed data was performed using the AMIRA $^{\circledR}$ software from TGS Europe (France). When processing raw data for 3D reconstructed image, different steps are required:

- Visualisation of several slices (in the 3D space) of raw image to check the quality of data and reject regions where the signal-to-noise ratio is not optimal. Due to the large amount of data (about 4 Gbytes) and consequently the long computation time, the volume of interest in the example presented here was reduced to a suitable size. The image volume was typically reduced to 400 x 400 x 268 voxels.

- $\quad$ Use of filters in order to further reduce the noise. Here, we used an "Edge-Preserving Smoothing" filter [9]. This filter smoothes the image without smearing out the edges because the filter process is stopped in the vicinity of edges. Thus, edges, which qualify the interfaces between different phases (i.e. solid and water), are preserved.

- Extraction of the surface of the interfaces between solid material and water in the entire volume. This surface, called the "isosurface", encloses all parts of the volume of interest that are brighter than some user-defined threshold. In the case of the image presented below, the threshold was set to 120 .

- Visualisation of the 3D membrane reconstruction. 
All necessary information about the 3D reconstruction visualisation can be found in the Amira manual [9]. In this first attempt to apply SR $\mu \mathrm{CT}$ to the characterisation of a microfiltration membrane, we did not seek to optimize or rationalize the segmentation / extraction procedures. Filter parameters and extraction parameters were set manually. Rationalization of the image processing and analysis should be undertaken in a further step.

\section{Results and discussion}

\subsection{Morphological characterization}

Figure 1(a) shows a projection of 1072 two-dimensional radiographs obtained by SR microtomography of a sample of wet hollow-fibre microfiltration membrane. The difference in absorption coefficients of the polymer phase and water phase clearly discriminates between white areas corresponding to the polymer solid phase and grey areas corresponding to the water phase. The white to grey shading that appears in the different parts of the image corresponds to different porosities, from the most dense (white) to the most porous (grey). Some parts of the fibre were not properly acquired during the experiment as can be seen on the upper left of the image. So we selected the bottom part of the fibre on the image of figure 1(a). White and grey stripes or rings are also visible on this figure. These are artefacts which will be removed afterwards.

As illustrated on Figure 1, SR computed tomography enables a complete visualisation of the cross-section of the membrane without preparation or cutting of the material. Here, we can clearly see that the morphology of the porous network varies considerably within the sample

thickness. We can clearly see four regions with very different structures. From the centre of the fibre to the outside, a first region (labelled (1) on figure 1) appears as a dense polymeric region. We expect pores to be present in the area, otherwise there would be no permeation through the structure. Since, in our conditions, the resolution was limited to 0.7 microns, we 
concluded that all the pores present in this region were necessarily smaller than 0.7 microns. We defined this region as a porous polymeric phase with pores smaller than $0.7 \mu \mathrm{m}$.

The region labelled 2 differs markedly from the previous one since it consists of a large zone of macrovoids. This region corresponds to a volume where the coalescence of the polymerpoor phase occurred during the membrane elaboration and, consequently, large pores were formed. The walls of the pore formed from a porous polymer that appears to be the same as the porous polymeric phase observed in region (1) as demonstrated from SEM observations (see insert in Figure 1).

From this transversal view, the anisotropic structure of pores in this region shows a finger-like pattern. In some parts, the length of the macrovoids is similar to the thickness of this region while, in other parts, the structure consists of smaller macrovoids connected in a necklace arrangement. Note that, as this image corresponds to a slice $0.7 \mu \mathrm{m}$ thick, some discontinuities may also appear in the structure. But it should be borne in mind that only a limited number of 2D projections have been visualized here.

The region labelled 3 is again a zone of macrovoids, the structure of which is also finger-like. However, this region is much thinner than the previous one. Compared to those of region 2, macrovoids in this region are small and numerous. The two distinct regions where macrovoids are visible are separated by a white "skin", indicating a distinct morphology of the structure at the frontier between the two zones. Finally, at the outer part of the structure, a fourth region, quite similar to region 1, i.e. visible here as a polymer-rich region, can be observed.

Figure 1(b) shows a perspective display of three 2D radiographs taken at different lengths of the hollow fibre, while Figure 1(c) shows a representation of radial sections at a given length. As shown, information concerning radial and longitudinal variations is readily available from the SR tomographic experiments and does not require any preparation or cutting of the fibre. A very large number of observations would be required to obtain approximately the same 
result with an SEM technique. Complete information about the three-dimensional structure of the sample is certainly the most outstanding advantage of this technique.

Using figure 1-a) and figure 2, we compare synchrotron radiation (SR) radiography of a wet fibre with an image of a dry fibre obtained by scanning electron microscopy (SEM). At this stage, the SR $\mu \mathrm{CT}$ image is not modified by any mathematical algorithm. For the SR $\mu \mathrm{CT}$ image, a slice of about $0.7 \mu \mathrm{m}$ of the membrane is presented whereas, in the SEM image, the fibre was cut transversally. It is well known that the drying, freezing, breaking and metal coating procedures used for SEM imaging can affect the morphological properties of a membrane. Drying is known to affect the external shape of the membrane, so its internal structure could also be affected. During breaking, the membrane structure could be affected by plastic deformation. Debris resulting from breakage can be observed on the SEM image. One of the practical advantages of SR $\mu \mathrm{CT}$ is that it allows direct observation of the fibre in conditions close to operational running. While the correspondence between the two figures is obvious, it should not be forgotten that they actually correspond to very different information. For the SR $\mu \mathrm{CT}$ image, the fibre is not sectioned and the picture shown is a view across the wet membrane at a given length. The observed structure is not affected by the sample preparation contrary to that obtained by SEM. The SR $\mu C T$ image of the figure 1-a) is one of the 1024 images obtained in one experiment (corresponding to about $720 \mu \mathrm{m}$ of fibre) whereas the SEM image is one image obtained in one experiment (corresponding to few $\mu \mathrm{m}$ ). Using $\mathrm{SR} \mu \mathrm{CT}$, any 3D plan can be visualized in one experiment.

A more complete rendition of the 3D structure is presented in Figure 3. This image represents a volume of 595 x 456 x 268 voxels or 420 x 320 x $190 \mu \mathrm{m}$ reconstructed from a small part of the raw data presented above. The inner skin is situated on the upper side of the volume in the xy plane and the outer skin on the lower side of the volume. 
The process used to get this image consisted in a) using filters in order to reduce the noise in the raw data, b) performing a segmentation of the data. It appears that the grey-value range from 120 to 255 visually corresponds to the membrane material and the grey-value range from 0 to 119 corresponds to water. So the "isosurfaces" which enclose all voxels of the volume of interest that are brighter than the threshold (here 120) could be extracted. The surface thus represents the interface between solidified polymer-rich regions and pores filled with water. This image shows that this microfiltration membrane is actually a complex threedimensional bi-continuum of interconnected pores. The four regions previously identified are also clear on this image, which shows that large pores are present in regions 3 and 2, with a more complex honeycomb structure. The thickness of regions 1 and 4 (inner and outer skins) is between $2.8 \mu \mathrm{m}$ and $5 \mu \mathrm{m}$ for the inner layer and between $1.6 \mu \mathrm{m}$ and $2 \mu \mathrm{m}$ for the outer layer.

The external and internal visualisation of the reconstructed volume can be observed on movie 1 and movie 2 respectively (see additional material on JMS Web site).

Figure 4 shows successive 2D sectional sections taken along the $\mathrm{z}$ axis across the reconstructed volume presented in Figure 3. These sections give good information about the microstructure and connectivity of the pores, which is very important with regard to transport phenomena. Sections 1) to 4) are included in the region labelled 2 (large macrovoids region) whereas section 5) is included in region labelled 3 (smaller macrovoids region). Comparing these $2 \mathrm{D}$ sectional views indicates that the structure in the xy plane is similar within regions 2 and 3. A rough estimate of the mean pore size in each of the five different sections is given in Table 2. The same observation could be made for pores between the outer skin and the outer interface. To our knowledge, it is the first time that the structure of a membrane has been observed in the radial direction. 
These measurements indicate that the mean pore size tends to increase along the $\mathrm{z}$ axis in region (2) and decrease along the $\mathrm{z}$ axis in region (3). The observations of the region labelled 2 of the 3D reconstructed membrane (the same observation could be made in the region labelled 3) show that:

- $\quad$ the geometry of pores is roughly hexagonal or pentagonal. The pores are organised in a honeycomb structure.

- the pores are roughly conical from the inner skin to the inner interface (near slice number 4) with diameters between 1 and $140 \mu \mathrm{m}$ (assuming the local pore shape to be a cylinder) and the tortuosity is very close to 1.

- because of the conical shape of the pores, at a given point in the radial direction, two neighbouring pores merge to form a bigger pore. This can be seen on the image of section 3: the arrows indicate the disappearance of the wall between two pores.

- the pores of the inner skin that can be detected by the SR $\mu \mathrm{CT}$ are connected to the beginning the conical pore of the region labelled 2.

Figure 5 shows a filtered SR $\mu \mathrm{CT}$ radial image in region 2. We recognize, here, a typical structure resembling the structure of highly concentrated water-in-oil emulsion or dispersion, or in foam with low liquid content $[10,11,12]$ : a thin film between two liquid phases, plateau borders and vertices. The "water-in-oil-dispersion" type of structure might be obtained during the phase inversion with the high concentration of water corresponding to the final porosity of the membrane; here $80 \%$ (see below).

Movie 3 shows the structure of the membrane along the $\mathrm{z}$ axis (similar to figures 4 and 5) from the inner skin to the outer skin (see additional material on JMS web site). 
These observations are linked to the transport phenomena, phase separation and coalescence which occur during the decomposition and solidification of the polymer solution into the polymer-rich and polymer-poor phases. The overall phenomena could be described like this (see figure 6):

- Starting from a homogenous solution, a homogenous diffusion process begins, rapidly resulting in the formation of the skin by spinodal decomposition. The resulting skin can be seen as a dense surface with some pores.

- Diffusion of solvent and non-solvent then becomes heterogeneous and hindered. Relatively fast diffusion occurs through the pores and relatively slow diffusion through the dense part of the skin. Of course, this is an idealized point of view but it is not so unrealistic at pore-size scale. The result is that the diffusion fronts of the nonsolvent and the solvent are not parallel to the skin but are spatially dependent with a maximum of concentration located in the pores of the skin.

- At a given point inside the membrane, when the concentration of non-solvent transferred by diffusion is high enough, liquid/liquid phase separation occurs. The initial nuclei of this process (nucleation and growth) could well be located at the surface of the exit of the nascent skin's pores. So the interface between the polymerrich phase and the polymer-poor phase is located at the pore wall of the nascent skin. Then a water-in-oil dispersion forms at the pore surface of the nascent skin.

- $\quad$ The liquid/liquid phase separation continues from the exit of the nascent skin pore to the inside of the solution in the radial direction. The interface of the two liquids (and therefore of the future pore) is then oriented by the presence (or absence) of a subjacent pore and a "liquid channel" forms. The coalescence of two liquid channels gives a larger liquid channel.

- $\quad$ The liquid wall (polymer-rich phase) of the liquid channel evolves towards a gel phase then towards a solid phase with increasing polymer concentration. So, at a given point 
inside the membrane, the coalescence of the polymer-poor phase is stopped because the polymer-rich phase cannot move and the final structure of the pore is obtained. The solid phase forms a "kind" of membrane that separates the non-solvent and a non coagulated phase. This non coagulated phase will occur later in a secondary phase separation

- $\quad$ These different steps occur first at the surface of the nascent skin and progress towards the inside with the diffusion of solvent and non-solvent (in time and space).

So the 3D structure (taken at a given point of the 3D space) is the trace of the coalescence process that occurred when the coalescence stopped (taken at the same given point). It also indicates that the pores (and structure) grow from the inner (or outer) skin towards the centre of the membrane.

However, the presence and the position of a porous polymer phase at the interface between regions 2 and 3 raises questionings. It results from another phase inversion process that starts at the other interface of the hollow fibre. So this interface corresponds to the meeting of the two coalescence processes simultaneously occurring during membrane elaboration.

Why this interface is closer to the external skin than to the internal skin could be explained by the different conditions at the internal and external skins. For the internal skin, the coagulant medium is volume limited while the external coagulant medium is infinite and initially made up of pure water. Consequently, the diffusion of non-solvent will be less marked within the inner surface than the outer surface. The coagulation will be faster at the outside and the structure more quickly fixed. The position of this interface could thus be related to the kinetics of the processes. Of course, this description of the phenomena is only hypothetical and does not take account of the diffusion coefficient of the water and NMP, the 
thermodynamic properties or the physicochemical properties of the ternary systems. It needs to be confirmed by other experiments.

3D computed tomograms also provide the basis for the calculation of 3D mean local mass density to identify the porous polymer phase and pores and thus calculate a porosity scale (for pore sizes larger than the resolution) [13]. We calculated the apparent porosity of different regions of the membrane from the number of voxels of the volume under consideration and the number of voxels assigned to the liquid lying in this volume by means of the following equation.

$\varepsilon=\frac{\text { number of voxels of liquid }}{\text { number of voxels in the considered volume }}$

Table 2 shows the estimated porosity of the different slices. The porosity of the membrane does not change across the membrane while the pore size changes tremendously. More specifically, the grey value is linked to the apparent density of the matter. By using suitable calibration (not performed here), the porosity of the material of a voxel could be calculated $[5,14]$. From the SEM observations (see figure 7), we can see that the pore walls of the macrovoid are also porous with pore size in the $0.1 \mu \mathrm{m}$ range. From the raw data (see figure 5), it can be deduced that the walls are also porous since the grey level varies from 255 (more dense) to 120 (more porous).

\subsection{Detection of defects in the skins}

Systematic investigations of the raw data in the regions corresponding to the polymer-rich phase (regions 1 and 4) revealed that local defects of large dimensions could be detected in both skins. Figure 8 shows a zoom on the upper part of the reconstructed volume presented in 
figure 3. On this figure, it is obvious that, at several places, the grey level of voxels is significantly lower than the threshold corresponding to a polymer-poor phase. It can also be observed that the series of defects have a specific orientation along the spin axis. An order of magnitude can be given for these defects: around 3 microns.

Figure 9 presents a 2D section in the xz plane of one of the defects shown before. The grey parts represent the polymer-rich phase and the white parts the voids. From this figure it is obvious that these defects are actually a continuation of the macrovoids present in regions (2) and (3).

\section{Conclusion}

In this paper, we have shown how the three-dimensional morphological properties of a PVDF hollow fibre can be investigated thanks to the new potentialities associated with X-ray synchrotron microcomputed tomography. From these first results, it is clear that SR $\mu \mathrm{CT}$ combined with 3D reconstruction allows a new type of observation of the structure of polymeric membranes.

3D morphological data of a PvDF hollow fibre microfiltration membrane prepared by phase inversion obtained from the ID19 line at the ESRF are presented. The membrane actually appears as a complex three-dimensional bi-continuum of interconnected pores. Within the hollow fibre structure, different regions with variable thicknesses and pore size distributions are identified and well characterized. Transversal views show the anisotropic finger-like structure of pores, whereas longitudinal sections reveal a honeycomb structure which resembles the structure of highly concentrated water-in-oil emulsion or dispersion. This typical structure might be obtained during the phase inversion process. How the phase inversion process may result in these morphologies was finally discussed. 
In the future, the increase in image resolution in progress at ESRF (down to the $100 \mathrm{~nm}$ range) will make SR $\mu \mathrm{CT}$ even more attractive for membrane science.

Processing raw data from such 3D images is a huge and complex task. In this first attempt, we have used basic steps and functions for the image processing. As mentioned, we used filters and parameters set manually. Rationalization and optimization of image processing should be undertaken in a further step.

\section{References}

[1] Phase field simulations of early stage structure formation during immersion precipitation of polymeric membranes in 2D and 3 D, B. Zhou, A.C. Powell, J. Membr. Sci. 268 (2006) $150-164$

[2] Strong effect of precursor preparation on the morphology of semicrystalline phase inversion poly(vinylidene fluoride) membranes, D.J.Lin, K. Beltsios, T.H. Young, Y.S. Jeng, L.P. Cheng, J. Membr. Sci. 274 (2006) 64-72.

[3] Chen, V., H. Li, and A.G. Fane, Non-invasive observation of synthetic membrane processes - a review of methods. J. Membr. Sci. 241(1) (2004) 23-44.

[4] Yeo, A., et al., Non-invasive observation of external and internal deposition during membrane filtration by X-ray microimaging (XMI). J. Membr. Sci. 250 (1-2) (2005)189193.

[5] J. Baruchel, J.-Y.Buffière, E. Maire, P. Merle, G. Peix, X-ray tomography in material science, Hermes Science Publications, Paris, 2000.

[6] Menut, P., Rouch, J.C., Remigy, J.C. Elaboration de contacteurs à membrane : fabrication de fibres hydrophobes en PVDF et PVDF-HFP. in 9ème Colloque PROSETIA. 2004. Toulouse, France. 
[7] Baruchel, J., ID19 High-resolution Diffraction Topography Beamline, http://www.esrf.eu/UsersAndScience/Experiments/Imaging/ID19/ (03/28/2007), European Synchrotron Radiation Facility.

[8] http://www.esrf.eu/UsersAndScience/Experiments/Imaging/ID19/BeamlineDescription/De tectors/Frelon_intro $(03 / 28 / 2007)$.

[9] http://www.amiravis.com/amira40-PDFindex.html (03/28/07).

[10] V.G. Babak, M.J. Stebe, Highly concentrated emulsions: Physicochemical principles of formulation, J. Dispersion Sci. Tech. 23(1-3) (2002), 1 -22.

[11] H.-J. Butt, K. Graf, M. Kappl, Physics and Chemistry of Interfaces, Wiley \& Sons, Weinheim (2003).

[12] Stephan A. Koehler, Sascha Hilgenfeldt, and Howard A. Stone, A Generalized View of Foam Drainage: Experiment and Theory, Langmuir 2000, 16, 6327-6341.

[13] A. Elmoutaouakkil1, G. Fuchs, P. Bergounhon, R. Peres and F. Peyrin, Threedimensional quantitative analysis of polymer foams from synchrotron radiation x-ray microtomography. Journal of Physics D-Applied Physics, 2003. 36(10A): p. A37-A43.

[14] H. Tauda,T, R. Martinez-Angelesa, J.F. Parrotb, L. Hernandez-Escobedoa, Porosity estimation method by X-ray computed tomography, Journal of Petroleum Science and Engineering 47 (2005) 209- 217.

\section{List of figures}

Figure 1: Images from SR $\mu \mathrm{CT}$ observation of PvDF co HFP HF a) An example of one slice, b) 0, 137, 268 slices the observed volume. c) xy, yz and xz slices of the same observed volume. Dimension of the observed volume (i.e. the box) are 2048 x 1950 x 268 voxels. 
Figure 2: SEM photograph of PvDF co HFP HF. external diameter: $1.41 \mathrm{~mm}$, internal diameter: $0.78 \mathrm{~mm}$.

Figure 3: 3D reconstructed volume of a part of PvDF co HFP HF from SR $\mu$ CT. Dimensions of the observed volume (i.e. the box) are 595 x 456 x 268 voxels or 420 x 320 x $190 \mu \mathrm{m}$.

Figure 4: Position and structure of the different slices considered. Dimensions of the observed volume (i.e. the box) are 595 x 456 x 268 voxels or 420 x 320 x $190 \mu \mathrm{m}$.

Note there is still some noise at the internal and the external surface of the 3D reconstructed membrane.

Figure 5: Honeycomb structure obtained from filtered SR $\mu \mathrm{CT}$ image of the region labelled 2. Dimensions of the image (i.e. the box) are 498 x 293 voxels or 349 x $224 \mu \mathrm{m}$.

Figure 6: Schematic representation of the formation of the pores.

Figure 7: SEM photograph of a macrovoid.

Figure 8: 3D reconstructed slice of the membrane internal skin with a defect: a) surface view, b) axial view. Dimensions of the observed volume are 39 x 32 x 13 voxels or 27.3 x 22.4 x $9.1 \mu \mathrm{m}$.

Figure 9: Cutting plane of the defect of figure 6: profiles of a) the larger dimension of the defect and b) of the smaller dimension of the defect. 


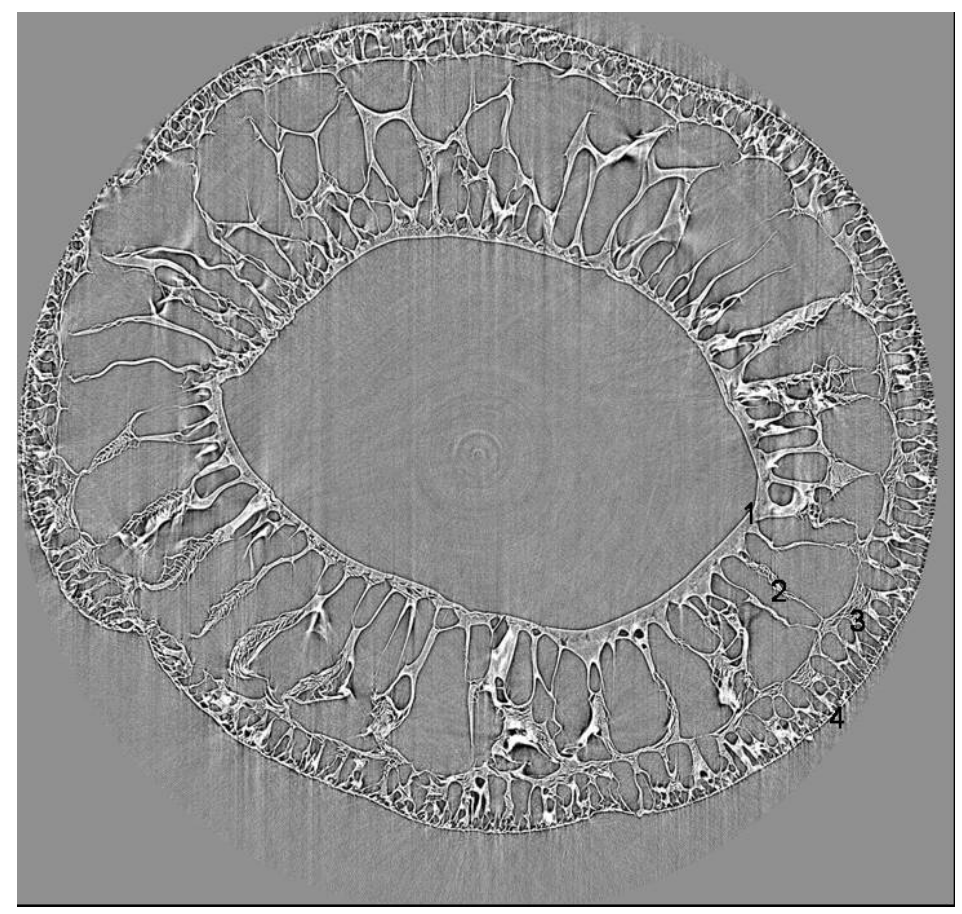

Figure 1 a)

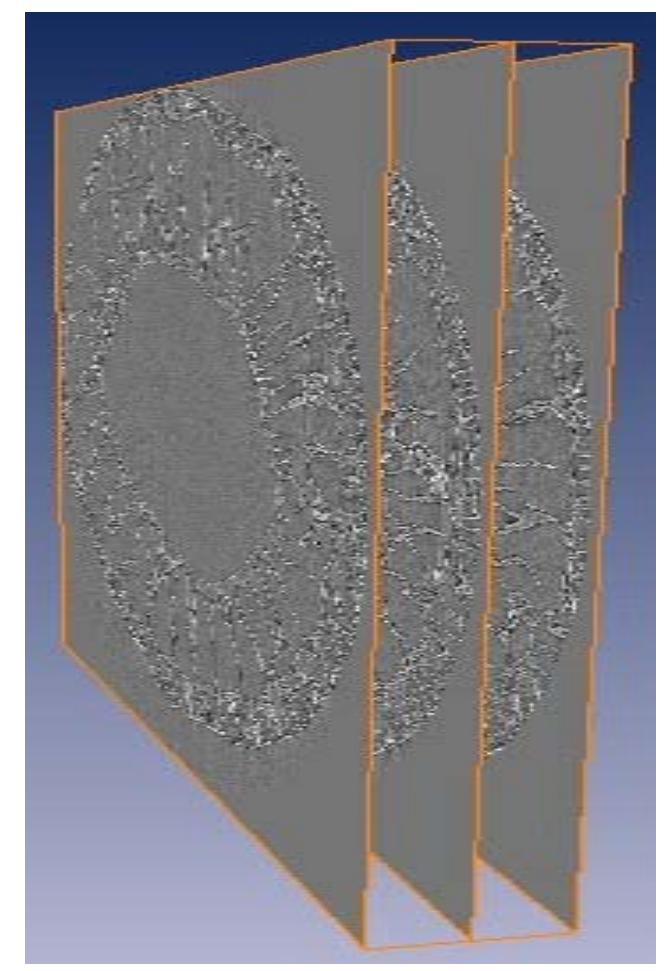

Figure 1 b) 


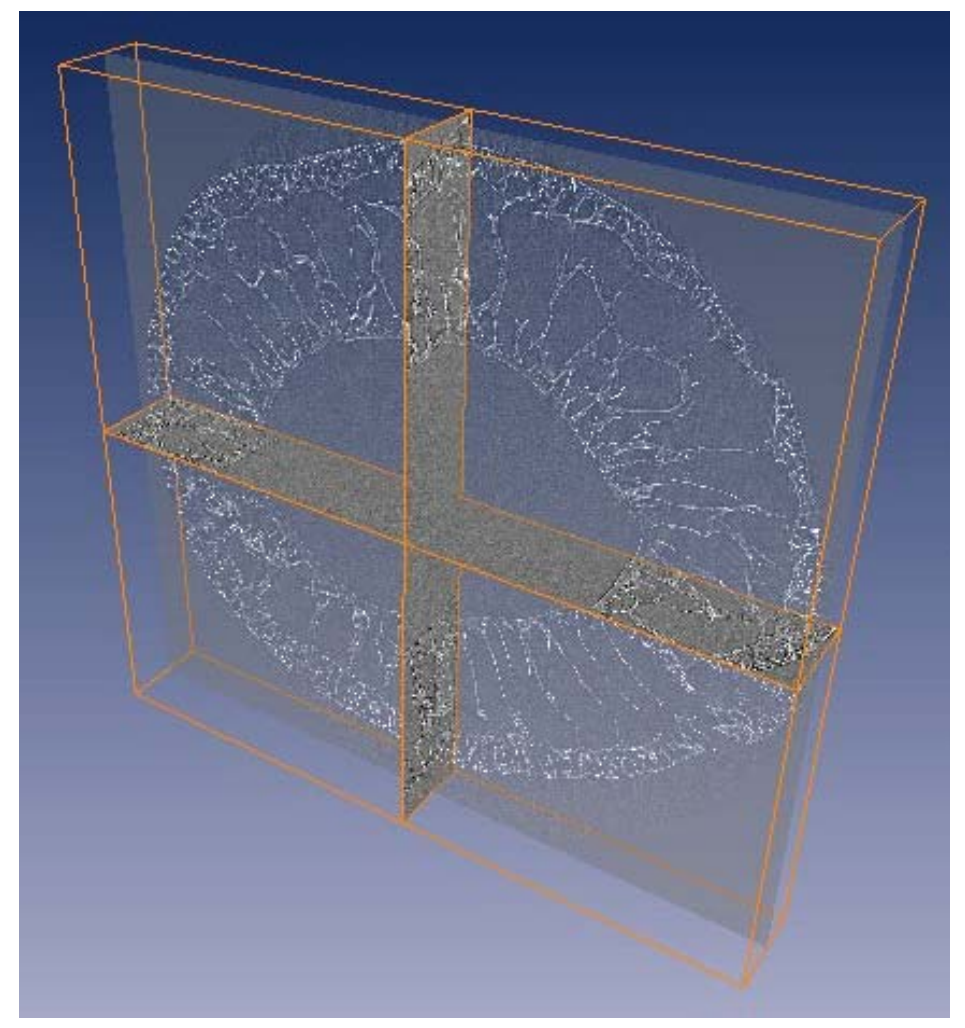

Figure 1 c)

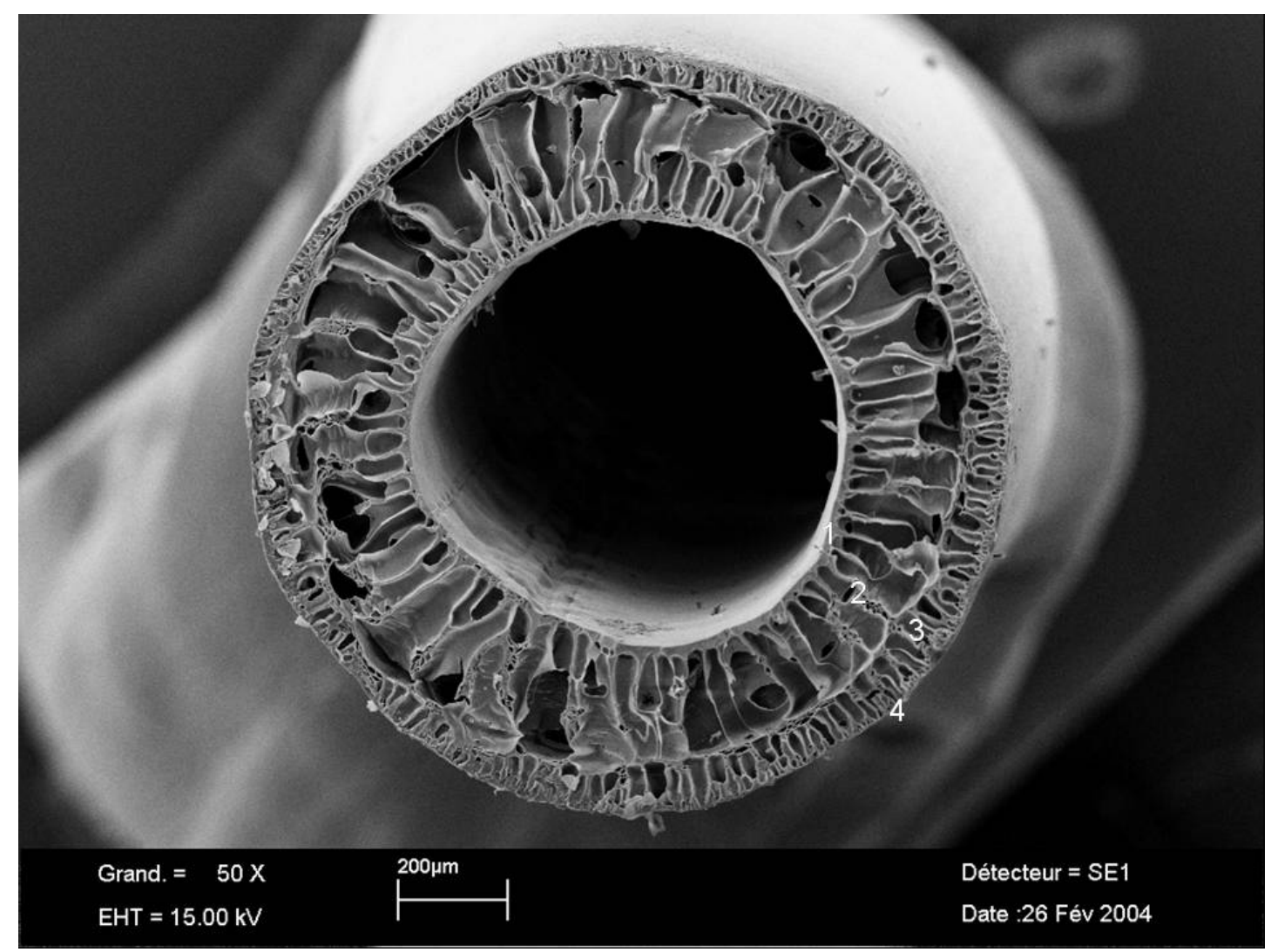

Figure 2 


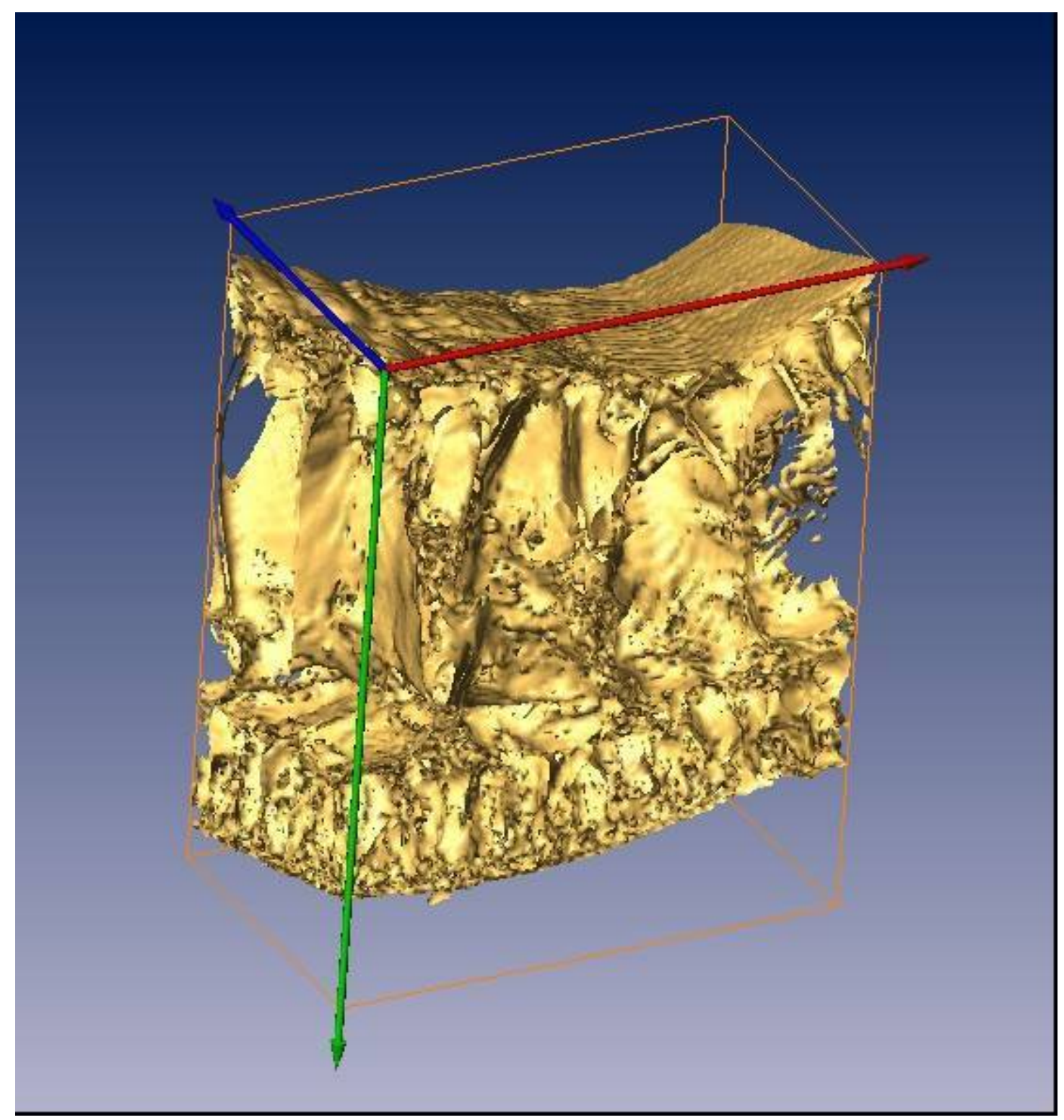

Figure 3 


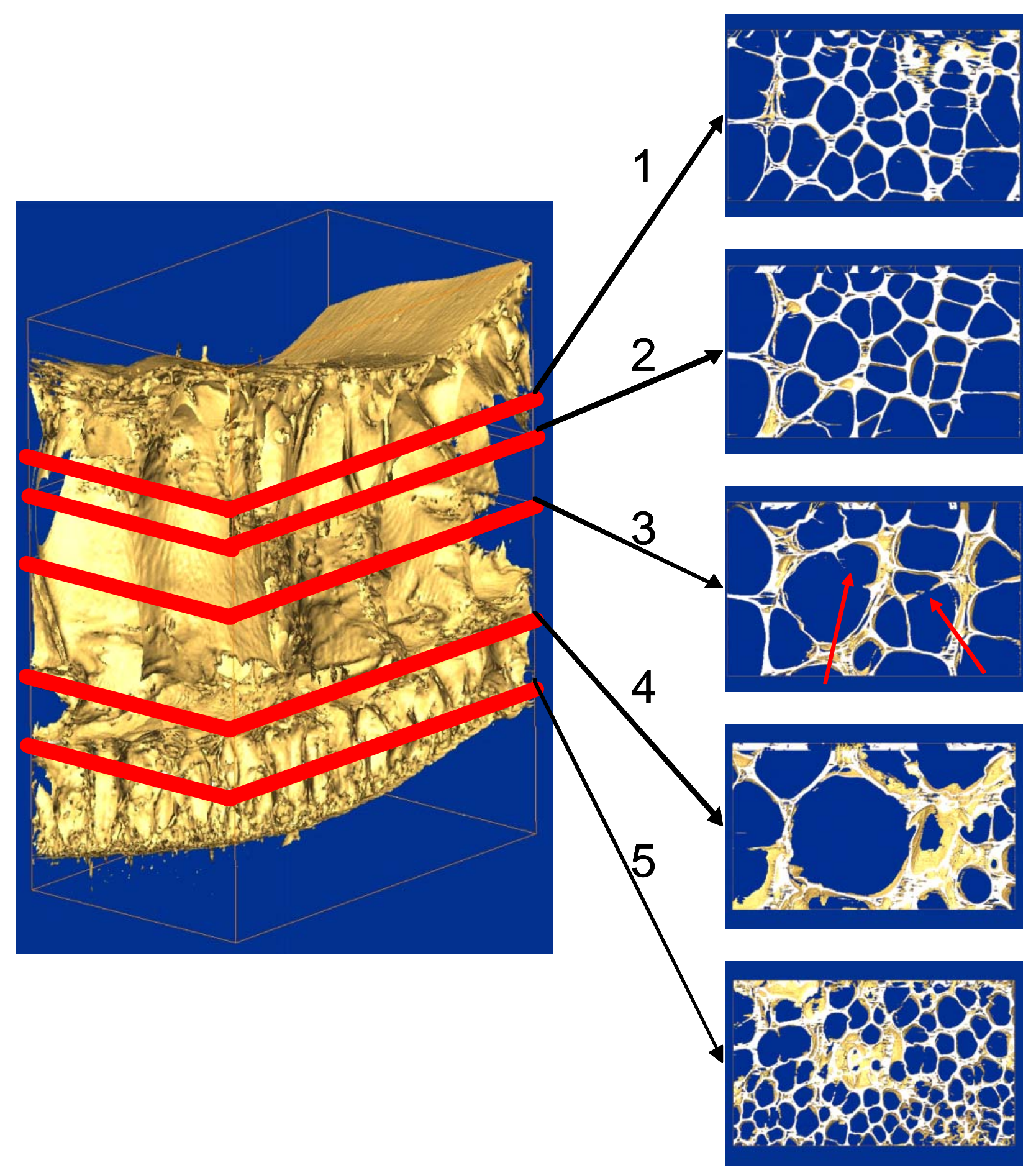

FIGURE 4 


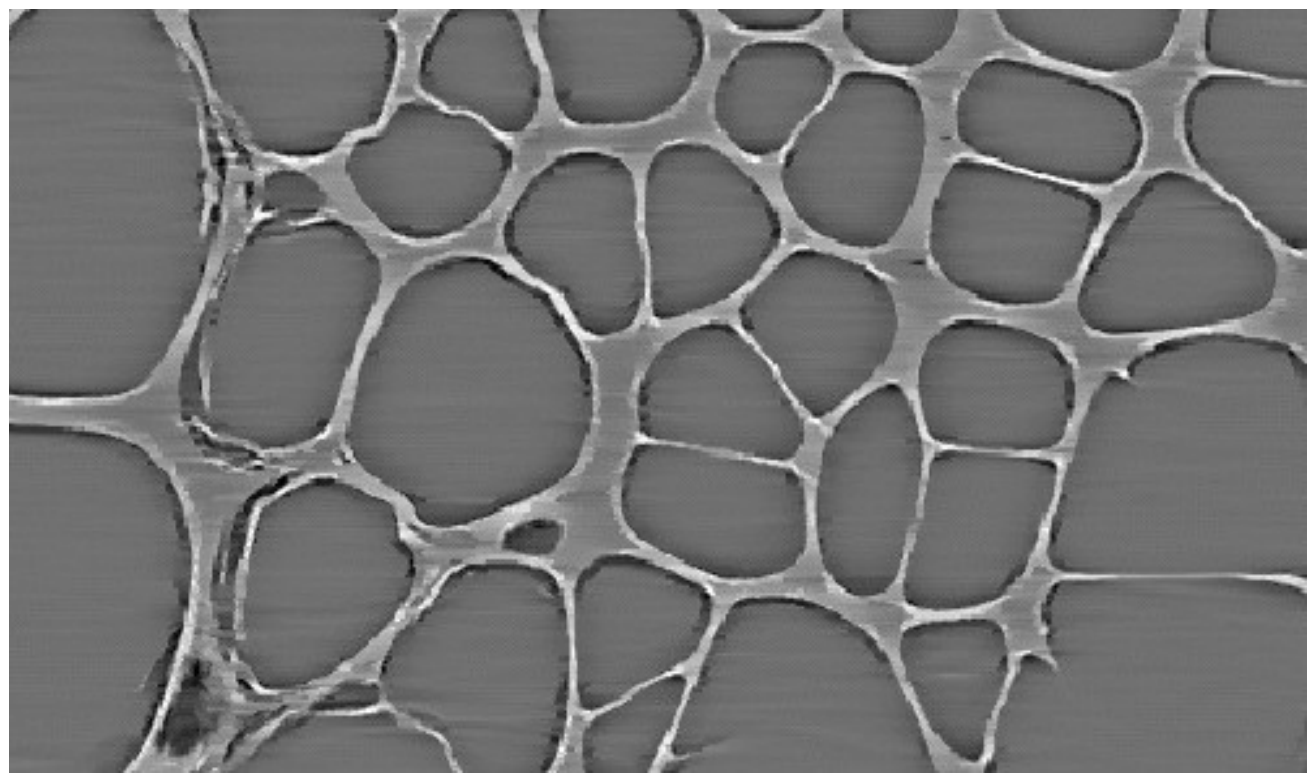

\section{Figure 5}

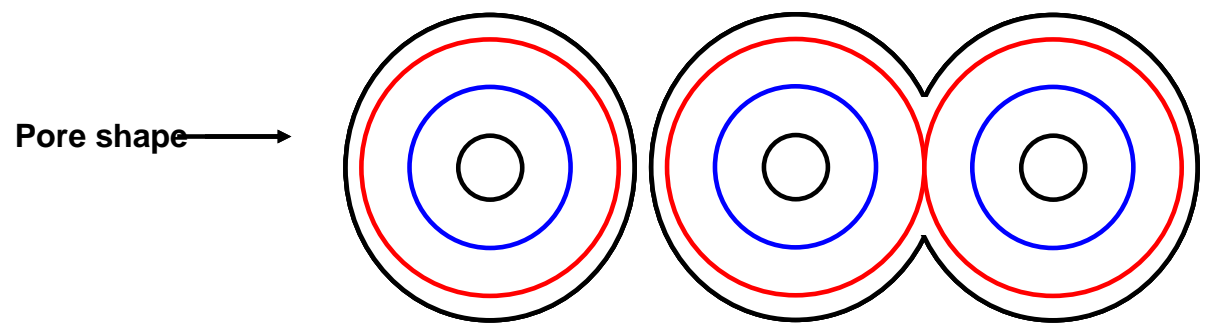

Front of phase separation

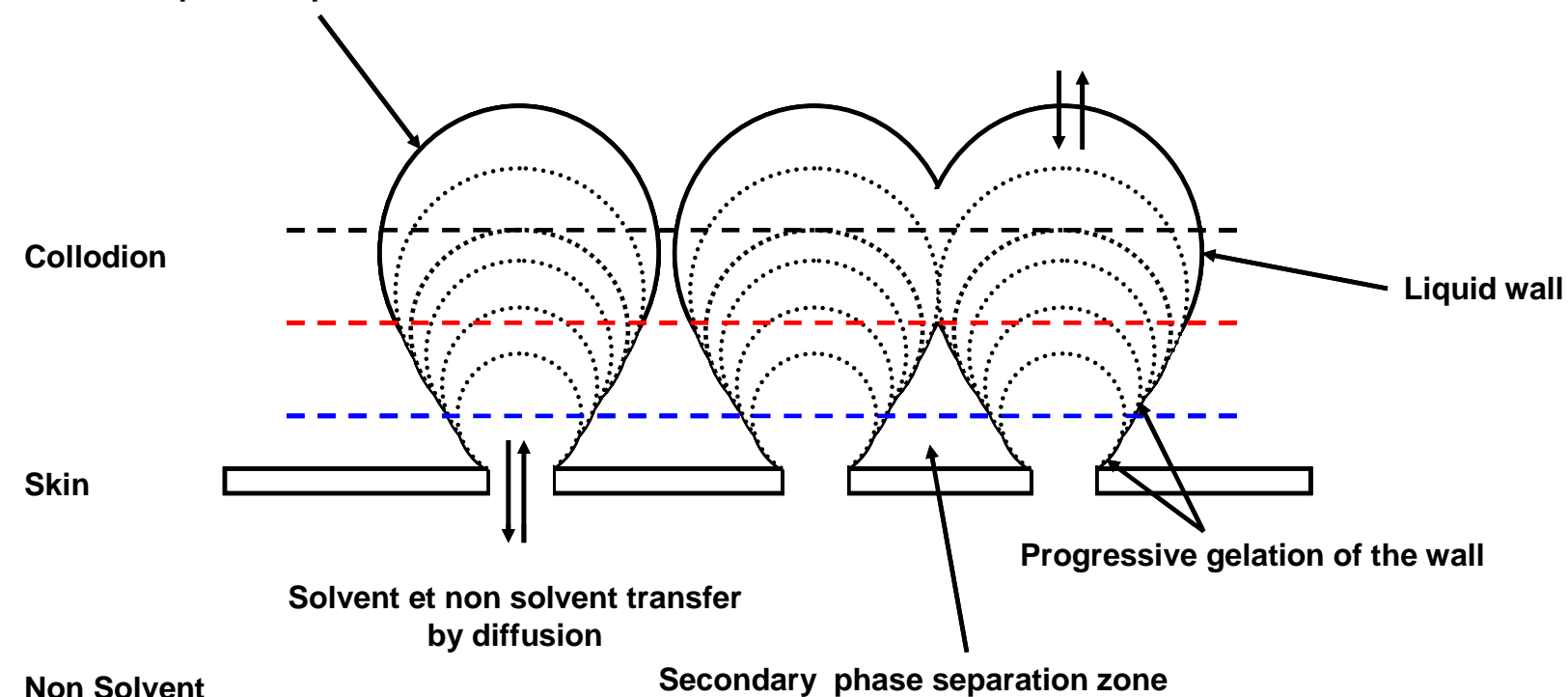

Figure 6 


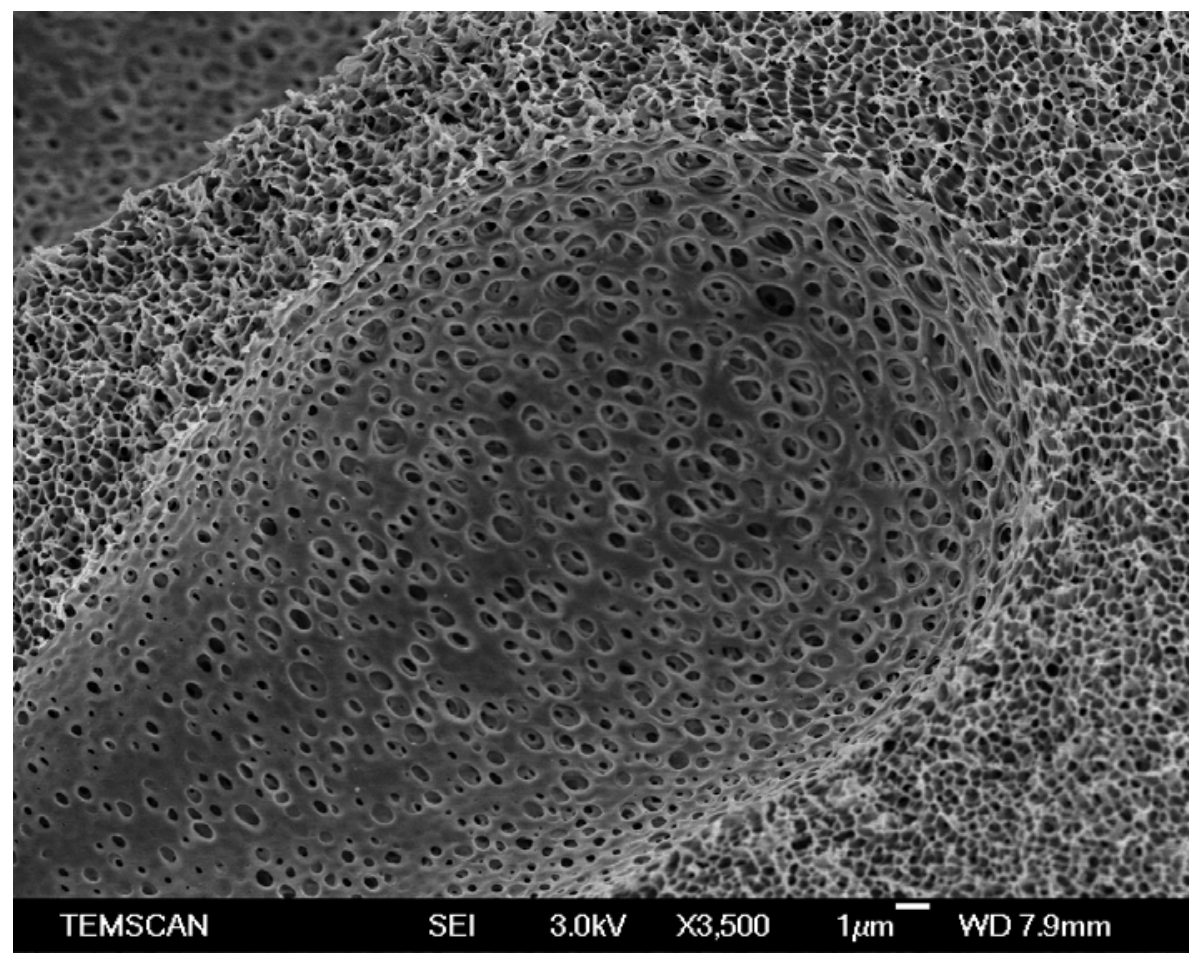

Figure 7 
Figure 8 a)
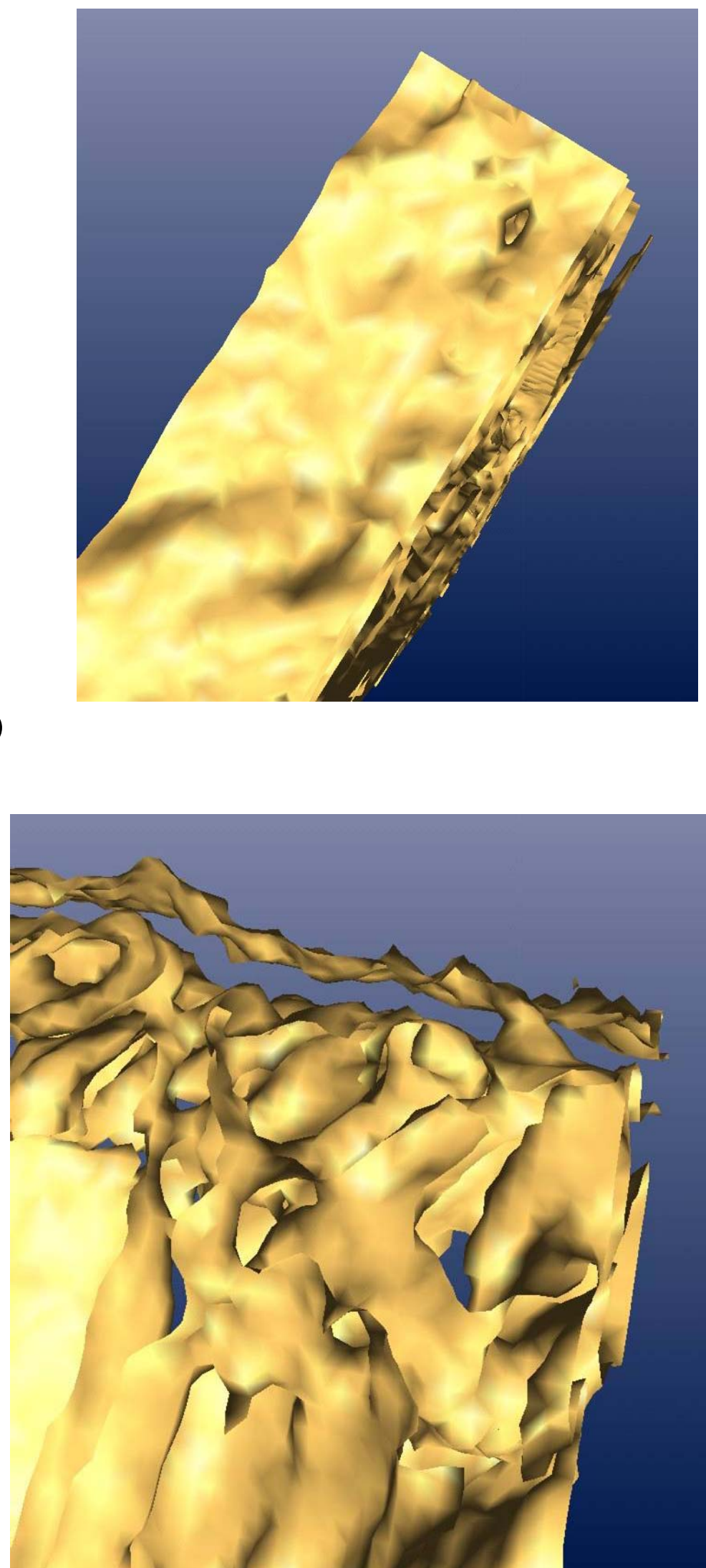

Figure 8 b) 


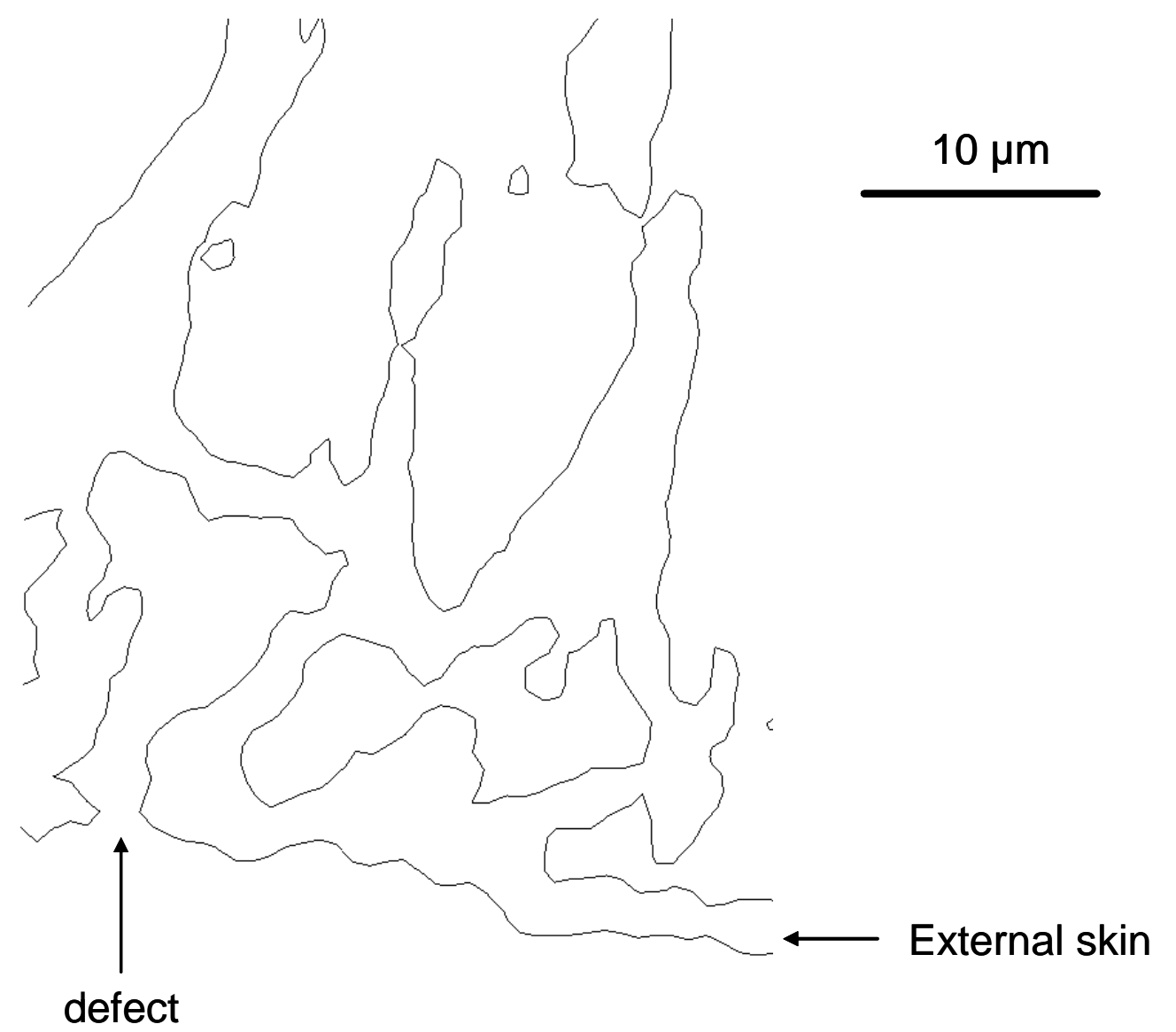

FIGURE 9 a)

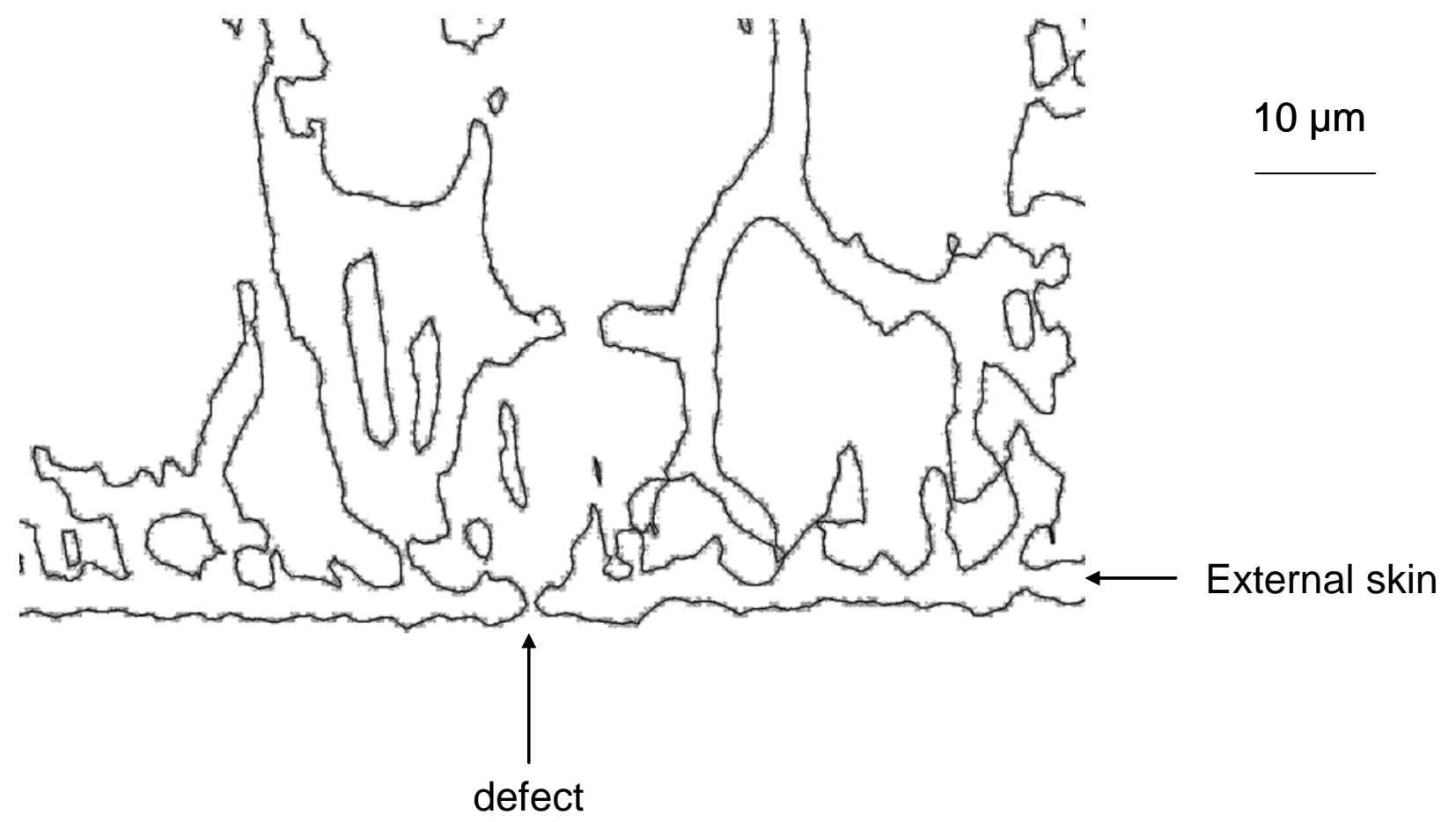

FIGURE 9 b) 
Table 1: spinning conditions and collodion composition.

Table 2: porosity and pore diameter range of different slices in the thickness of the membrane. 


\section{Table 1}

Collodion composition

\begin{tabular}{|c|c|c|}
\hline Product & Supplier & Mass fraction \\
\hline $\begin{array}{c}\text { PvDf-co HFP } \\
\text { Solef 21216/1001C }\end{array}$ & Solvay Belgium & $18 \%$ \\
\hline LiCl & Aldrich France & $6 \%$ \\
\hline Water & Reverse osmosis water & $2.4 \%$ \\
\hline DMAc & Aldrich France & $73.6 \%$ \\
\hline
\end{tabular}

Spinning conditions

\begin{tabular}{|l|l|}
\hline Air gap & $500 \mathrm{~mm}$ \\
\hline Bore liquid & Tap water \\
\hline Coagulation bath & Tap water \\
\hline Spinning rate & $3.4 \mathrm{~m} \cdot \mathrm{min}^{-1}$ \\
\hline Temperature & $32^{\circ} \mathrm{C}$ \\
\hline
\end{tabular}

\section{Table 2}

\begin{tabular}{|l|l|l|}
\hline Slice & Porosity & Pore diameter range \\
\hline Internal skin & $24^{* * *}$ & $0 *-13^{* *} \mu \mathrm{m}$ \\
\hline 1 & 77.8 & $7-72 \mu \mathrm{m}$ \\
\hline 2 & 82.4 & $23-80 \mu \mathrm{m}$ \\
\hline 3 & 83.6 & $30-90 \mu \mathrm{m}$ \\
\hline Internal interface & $65^{* * *}$ & $0 *-20 \mu \mathrm{m}$ \\
\hline 4 & 80.0 & $35-140 \mu \mathrm{m}$ \\
\hline 5 & 70.0 & $18-62 \mu \mathrm{m}$ \\
\hline External skin & $50^{* * *}$ & $0^{*}-4 \mu \mathrm{m}$ \\
\hline
\end{tabular}

* Below the resolution

** Defect

*** Roughly estimated 